\title{
Substrate thermal conductivity effect on heat dissipation and lifetime improvement of organic light-emitting diodes
}

\author{
Seungjun Chung, ${ }^{1}$ Jae-Hyun Lee, ${ }^{2}$ Jaewook Jeong, ${ }^{1}$ Jang-Joo Kim, ${ }^{2}$ and \\ Yongtaek Hong ${ }^{1, a)}$ \\ ${ }^{1}$ Department of Electrical Engineering and Computer Science, Inter-University Semiconductor Research \\ Center, Seoul National University, San 56-1 Sillim-9-Dong, Gwanak-Gu, Seoul 151-744, Republic \\ of Korea \\ ${ }^{2}$ Department of Material Science and Engineering, Center for OLED, Seoul National University, San 56-1 \\ Sillim-9-Dong, Gwanak-Gu, Seoul 151-744, Republic of Korea
}

(Received 30 September 2008; accepted 14 May 2009; published online 22 June 2009)

\begin{abstract}
We report substrate thermal conductivity effect on heat dissipation and lifetime improvement of organic light-emitting diodes (OLEDs). Heat dissipation behavior of top-emission OLEDs fabricated on silicon, glass, and planarized stainless steel substrates was measured by using an infrared camera. Peak temperature measured from the backside of each substrate was saturated to be $21.4,64.5$, and $40.5^{\circ} \mathrm{C}, 180 \mathrm{~s}$ after the OLED was operated at luminance of $10000 \mathrm{~cd} / \mathrm{m}^{2}$ and $80 \%$ luminance lifetime was about 198, 31, and 96 h, respectively. Efficient heat dissipation through the highly thermally conductive substrates reduced temperature increase, resulting in much improved OLED lifetime. (C) 2009 American Institute of Physics. [DOI: 10.1063/1.3154557]
\end{abstract}

Thermal stress and degradation of organic light-emitting diodes (OLEDs) has been widely studied ${ }^{1-3}$ because it is well known that luminance and lifetime decreases for OLEDs operated at elevated temperatures. ${ }^{4-7}$ It has been also reported that localized joule heating can degrade brightness homogeneity and this effect becomes worse as device temperature increases with operation time. ${ }^{8}$ Therefore, in order to further improve operational lifetime, it is crucial to efficiently dissipate heat generated inside the devices.

Effective heat-sinking can be obtained through substrate if the substrate has good thermal conductivity as in the case of silicon substrates $(150 \mathrm{~W} / \mathrm{m} \cdot \mathrm{K})$ in microelectronics applications. ${ }^{9}$ However, glass substrate that has been widely used for OLED display and lighting applications typically has low thermal conductivity $(1 \mathrm{~W} / \mathrm{m} \cdot \mathrm{K})$, showing poor heat sinking. It was reported that device temperature can increase up to as high as $86{ }^{\circ} \mathrm{C}$ for OLEDs on glass substrates. ${ }^{1,4}$ Although silicon substrate is used for OLED microdisplays, ${ }^{10}$ where it can act as a good heat sinker, there is scaling-up limitation for large-area applications.

Stainless steel (SUS) substrate can be a good candidate due to its relatively high thermal conductivity $(16 \mathrm{~W} / \mathrm{m} \cdot \mathrm{K})$. Although SUS substrate has been widely used for OLED displays ${ }^{11-14}$ and they can be key element for flexible OLED lighting applications, there are few reports on their efficient heat dissipation for potential OLED lifetime improvement. Therefore, in this paper, we compared heat dissipation behaviors of top-emission OLEDs (TEOLEDs) fabricated on glass (TEOLED-g), and SUS (TEOLED-SUS) substrates. We also fabricated TEOLEDs on silicon substrates (TEOLED-s) for reference. Thermal distribution and corresponding OLED lifetime effects were analyzed for these devices.

We used intrinsic silicon wafer with $200 \mathrm{~nm}$ thick thermal oxide, Corning Eagle ${ }^{2000 \mathrm{TM}}$ glass substrate, and 304 SUS

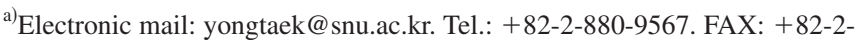
872-9714.
}

substrates. Thickness of each substrate was 550, 500, and $100 \mathrm{~mm}$, respectively, and their sizes were identical (1 $\times 1$ in. $^{2}$ ). Since surface roughness of the as-purchased SUS substrates was as high as $500 \mathrm{~nm}$ in peak-to-valley value, we planarized their surface with $3 \mu \mathrm{m}$ thick benzocyclobutene (BCB) (purchased from Dow Chemical Co., CYCLOTENE 3022-46) layer, and achieved surface roughness of $15 \pm 5 \mathrm{~nm}$. Surface roughness was measured by atomic force microscopy for five points of $5 \times 5 \mu \mathrm{m}^{2}$ scan area in $2 \times 2$ in. $^{2}$ samples. No additional surface treatment was applied to all substrates after cleaning them in ultrasonic bath of aceton and isoprophyl alcohol for 20 and $10 \mathrm{~min}$, respectively. For TEOLEDs, silver (Ag), molybdenum oxide $\left(\mathrm{MoO}_{3}\right), \quad \alpha$-napthylphenylbiphenyl $\quad$ (NPB), $\quad$ tris-(8hydroxyquinoline) aluminum $\left(\mathrm{Alq}_{3}\right)$, and ytterbium $(\mathrm{Yb}) / \mathrm{Ag}$ were used as materials of reflective anode $(300 \mathrm{~nm})$, hole injection layer $(4 \mathrm{~nm})$, hole transport layer $(54 \mathrm{~nm})$, electron transport and light-emitting layer $(53 \mathrm{~nm})$, and semitransparent cathode $(1 / 20 \mathrm{~nm})$, respectively. $\mathrm{Alq}_{3}$ index-matching layer $(45 \mathrm{~nm})$ was finally deposited. All the layers were sequentially deposited by thermal evaporation method without breaking vacuum and patterned through shadow masks to define light-emitting area of $3 \times 3 \mathrm{~mm}^{2}$. All fabricated devices were encapsulated by using glass caps with desiccant.

Figure 1 shows a schematic device structure and measured current density-voltage-luminance $(J-V-L)$ curves for the fabricated TEOLEDs. For thermal distribution and lifetime measurement, we selected bias conditions as summarized in Table I to produce $10000 \mathrm{~cd} / \mathrm{m}^{2}$ for each device. Thermal distribution and peak temperatures of the TEOLEDs were measured from backside of each substrate as shown in Fig. 1 by using an infrared (IR) thermal image camera (A60M from FLIR systems). IR thermal images were captured every $10 \mathrm{~s}$ right after the TEOLEDs were operated. Figure 2 shows selected images of thermal distribution changes with operation time. Temperatures in each image indicate peak temperatures of the imaging area. Thermal distribution is highly localized around the light-emitting area 


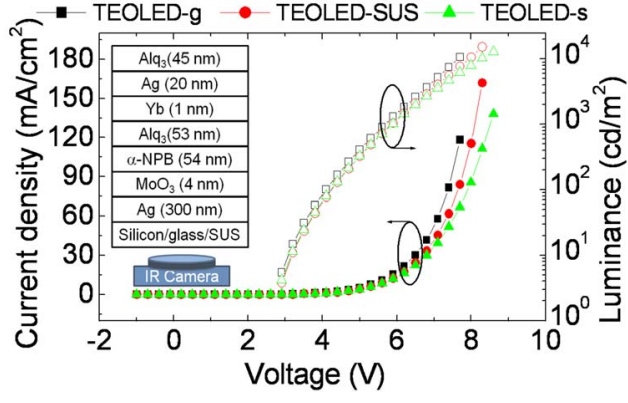

FIG. 1. (Color online) $J-V-L$ characteristics of TEOLEDs (schematic description of the TEOLED structure is included in inset).

for glass substrate while silicon and SUS substrates show less localization property. Especially, for silicon substrate, almost no localization was observed. Measured peak temperatures went up to $64.5,40.5$, and $21.4{ }^{\circ} \mathrm{C}$ for glass, SUS, and silicon substrates, respectively, indicating that heat generated inside the TEOLEDs was efficiently dissipated through the silicon and SUS substrates. It is noted that although the silicon oxide and $\mathrm{BCB}$ layers have poor thermal conductivities of 1 and $0.3 \mathrm{~W} / \mathrm{m} \cdot \mathrm{K},{ }^{15}$ respectively, they do not seem to block heat dissipation through the substrates because their thickness is very small in comparison with the substrates. In addition, since the light-emitting area is larger than their thickness, most heat generated in the TEOLEDs will be directly transferred to the substrate with little lateral diffusion. However, if the thickness of these layers becomes large compared to the substrate thickness, there will be a temperature gradient along the layers from anode to the substrates and relatively large lateral diffusion will be observed.

Figure 3 shows measured temperature distribution (symbols) over $25 \mathrm{~mm}$ across the light-emitting area of each TEOLED. Temperature increase $(\Delta T)$ with respect to initial temperatures of each substrate is plotted. TEOLED-g showed the highest peak temperature increase of about $45^{\circ} \mathrm{C}$ and more localized thermal distribution in comparison with TEOLED-SUS. TEOLED-s showed little temperature increase. We also performed thermal simulation for each substrate by using two-dimensional (2D) tool (named ATLAS) from SILVACO Inc., which numerically solves 2D lattice heat flow Eq. (1) with appropriate boundary condition parameters (2) as shown in the following equations to obtain temperature distribution in the substrate region:

$$
\begin{aligned}
& k \nabla^{2} T_{L}+H=0 \quad \text { (under steady state condition), } \\
& S=h \times\left(T_{L}-T_{\mathrm{ext}}\right) \quad \text { (boundary condition), }
\end{aligned}
$$

where $T_{L}, k, H, S, h$, and $T_{\text {ext }}$ are local lattice temperature, thermal conductivity, heat generation in OLED, heat flux per unit area, heat transfer coefficient, and external ambient temperature, respectively. The simulated results are shown as solid and dash lines, which correspond to different $h$ values.

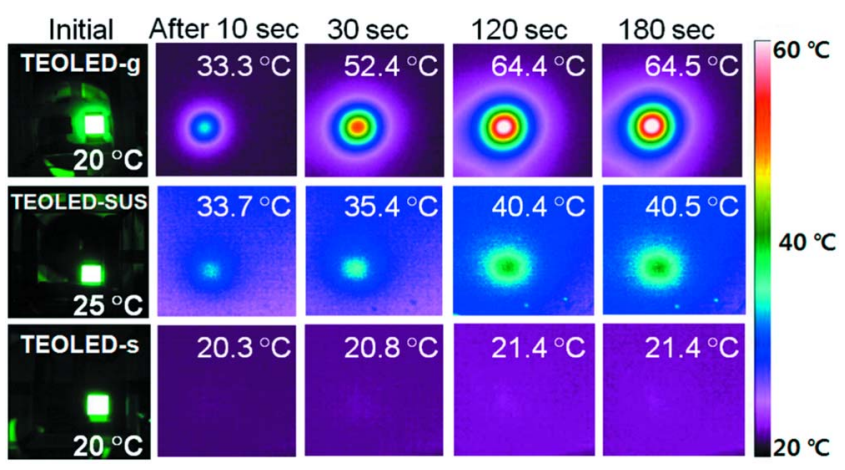

FIG. 2. (Color online) IR images of thermal distribution of TEOLED-g (top), TEOLED-SUS (middle), and TEOLED-s (bottom) $10000 \mathrm{~cd} / \mathrm{m}^{2}$ taken from the backside of the substrates.

Since it is difficult to accurately measure $h$ for each substrate, we used minimum and maximum values of $h$ as 0.005 (solid) and 0.01 (dash) $\mathrm{W} / \mathrm{cm}^{2} \mathrm{~K}$ in our simulation, assuming natural air convection at the backside of each substrate. We used an embedded poly(phenylenevinylele) (PPV)-based OLED module for convenience, and adjusted input electrical power $(82 \pm 1 \mathrm{~mW})$ and device efficiencies according to the measured data. Since light-emitting area is much larger than OLED thickness and we focused on thermal distribution through the substrates, it is believed that using PPV-based OLED module will not make much difference for our simulation purpose. In this $2 \mathrm{D}$ simulation, we can define lightemitting and substrate areas only in one dimension along device cross-section. Therefore, the simulation results may not fully represent three-dimensional thermal conduction behaviors, producing temperature distribution different from the measured data. This difference is more apparently observable for substrates with high thermal conductivity, such as SUS and silicon, for which the simulation results show higher $\Delta T$ than that of the measured results for both $h$ values. This is because only one-dimensional thermal conduction path is considered and thus, relatively smaller amount of heat conduction is considered for 2D simulation leading to more heat accumulation for the simulation result. However, for the substrate with low thermal conductivity such as glass, our simulation result showed a good agreement with the measured data, depending on $h$ values. Overall, both measured and simulated temperature distribution well represented tendency in temperature distribution change for substrates with different thermal conductivities.

In order to further investigate thermal stress effect on device lifetime, we performed lifetime test by applying constant current to the TEOLEDs. Current levels were adjusted so that each device produces initial luminance of $10000 \mathrm{~cd} / \mathrm{m}^{2}$. As shown in Table I, current variations for each device were within 5\%. Relative luminance change and operation voltage change $(\Delta V)$ are plotted with the operation

TABLE I. TEOLED experiment conditions for $10000 \mathrm{~cd} / \mathrm{m}^{2}$

\begin{tabular}{lcccccc}
\hline \hline & $\begin{array}{c}\text { Voltage } \\
(\mathrm{V})\end{array}$ & $\begin{array}{c}\text { Current } \\
(\mathrm{mA})\end{array}$ & $\begin{array}{c}\text { Current density } \\
\left(\mathrm{mA} / \mathrm{cm}^{2}\right)\end{array}$ & $\begin{array}{c}\text { Electrical input power } \\
(\mathrm{mW})\end{array}$ & $\begin{array}{c}\text { Luminous efficiency } \\
(\mathrm{cd} / \mathrm{A})\end{array}$ & $\begin{array}{c}\text { Power efficiency } \\
(\mathrm{lm} / \mathrm{W})\end{array}$ \\
\hline TEOLED-g & 7.7 & 10.7 & 118.32 & 82.4 & 9.03 & 3.70 \\
TEOLED-SUS & 8.0 & 10.4 & 115.56 & 83.2 & 9.34 & 3.67 \\
TEOLED-s & 8.3 & 10.3 & 111.55 & 85.5 & 9.34 \\
\hline \hline
\end{tabular}




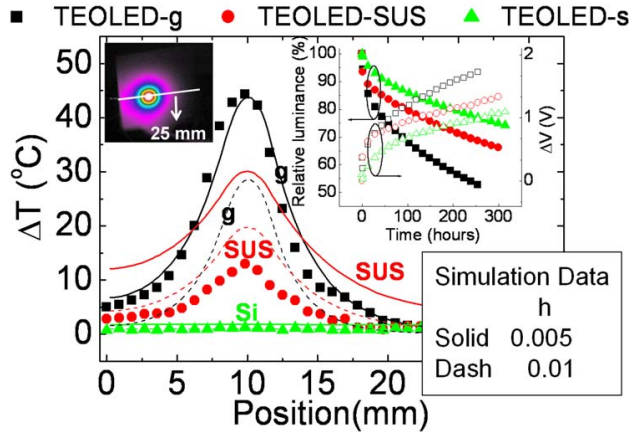

FIG. 3. (Color online) Temperature distribution across the localized heat spot: symbols and lines represent measured and simulated results, respectively. Solid and dash lines (g, SUS, Si) represent the simulated results for $h=0.005$ and $0.01 \mathrm{~W} / \mathrm{cm}^{2} \mathrm{~K}$ for each device. Lifetime measurement results are also included in inset.

time in the inset of Fig. 3. TEOLED-g and TEOLED-s showed the largest and smallest degradation for the same stress time, respectively. After $250 \mathrm{~h}$ of operation, $\Delta V$ values were about $1.7,1.2$, and $0.96 \mathrm{~V}$, for TEOLED-g, TEOLEDSUS, and TEOLED-s, respectively. The times of $80 \%$ luminance reduction from initial luminance were about 31,96 , and $198 \mathrm{~h}$, for TEOLED-g, TEOLED-SUS, and TEOLED-s, respectively.

Since OLED degradation is closely associated with surface roughness of bottom electrodes, we measured their surface roughness for all devices. All devices showed similar surface roughness of $2.7 \pm 1.2 \mathrm{~nm}$ in root mean square and $23 \pm 5 \mathrm{~nm}$ in peak-to-valley values, indicating surface roughness does not play a major role in lifetime difference. In addition, if we analyze the relationship between $80 \%$ lifetime and peak temperature values by using an equation of "lifetime $\sim \exp (\Delta E / k T)$ " that was reported by Aziz et al. ${ }^{16}$ for $50 \%$ lifetime of $a-\mathrm{NPB} / \mathrm{Alq}_{3}$ based OLEDs, we can obtain an activation energy $(\Delta E)$ of about $0.39 \mathrm{eV}$. This activation energy is related to the ionization potential difference between $a$-NPB and $\mathrm{Alq}_{3}$, which was reported as $0.35 \pm 0.15 \mathrm{eV}$. Our extracted activation energy is consistent with these values and degradation behavior seems to follow the operation temperature induced degradation behavior reported by Aziz et al. Therefore, it can be concluded that the lifetime improvement in our TEOLEDs is from effective heat dissipation through the substrates and corresponding smaller temperature increase.

In this letter, we analyzed heat dissipation of TEOLEDs through various substrates and corresponding lifetime behaviors. Efficient heat dissipation through silicon and planarized
SUS substrates further reduced peak temperature of the TEOLEDs, resulting in increased lifetime in comparison with glass substrates. It is noted that if highly efficient devices are used and corresponding peak temperature is little increased, substrate thermal conductivity effect on lifetime improvement can become smaller. However, since the OLED temperature increase is still an important issue in typical OLED display or lighting applications, highly thermally conductive substrates will be promising substrate candidates for such applications in terms of heat dissipation and corresponding lifetime improvement.

This work supported by the Korea Research Foundation Grant funded by the Korean Government (MOEHRD, Basic Research Promotion Fund) (Grant No. KRF-2008-331D00216). Authors Lee and Kim would like to acknowledge MKE through the Center for OLED for this work. Authors S. Chung, J. Jeong, and Y. Hong would like to thank InterUniversity Semiconductor Research Center for facility support and Professor Kwangseok Seo for BCB material and process support.

${ }^{1}$ X. Zhou, J. He, L. S. Liao, M. Lu, X. M. Ding, X. Y. Hou, X. M. Zhang, X. Q. He, and S. T. Lee, Adv. Mater. (Weinheim, Ger.) 12, 265 (2000).

${ }^{2}$ M. S. Xu and J. B. Xu, J. Phys. D 37, 1603 (2004).

${ }^{3}$ G. Nenna, G. Flaminio, T. Fasolino, C. Minarini, R. Miscioscia, D. Palumbo, and M. Pellegrino, Macromol. Symp. 247, 326 (2007).

${ }^{4}$ J. R. Sheats, H. Antoniadis, M. Hueschen, W. Leonard, J. Miller, R. Moon, D. Roitman, and A. Stocking, Science 273, 884 (1996).

${ }^{5}$ R. N. M. dos Anjos, H. Aziz, N.-X. Hu, and Z. D. Popovic, Org. Electron. 3, 9 (2002).

${ }^{6}$ G. Vamvounis, H. Aziz, N.-X. Hu, and Z. D. Popovic, Synth. Met. 143, 69 (2004)

${ }^{7}$ M. Ishii and Y. Taga, Appl. Phys. Lett. 80, 3430 (2002).

${ }^{8}$ C. Garditz, A. Winnacker, F. Schindler, and R. Paetzold, Appl. Phys. Lett. 90, 103506 (2007).

${ }^{9}$ D. B. Tuckerman and R. F. W. Pease, IEEE Electron Device Lett. 2, 126 (1981).

${ }^{10}$ G. B. Levy, W. Evans, J. Ebner, P. Farrell, M. Hufford, B. H. Allison, D. Wheeler, H. Lin, O. Prache, and E. Naviasky, IEEE J. Solid-State Circuits 37, 1879 (2002).

${ }^{11}$ J. H. Cheon, J. H. Choi, J. H. Hur, J. Jang, H. S. Shin, J. K. Jeong, Y. G. Mo, and H. K. Chung, IEEE Trans. Electron Devices 53, 1273 (2006).

${ }^{12}$ A. Kattamis, N. Giebink, I.-C. Cheng, S. R. Forrest, S. Wagner, Y. Hong, and V. Cannella, J. Soc. Inf. Disp. 15, 433 (2007).

${ }^{13}$ A. Chwang, R. Hewitt, K. Urbanik, J. Silvernail, K. Rajan, M. Hack, J. Brown, J. P. Lu, C. Shih, J. Ho, R. Street, T. Ramos, L. Moro, N. Rutherford, K. Tognoni, B. Anderson, and D. Huffman, SID Int. Symp. Digest Tech. Papers 37, 1858 (2006).

${ }^{14}$ T. K. Chuang, M. Traoccoli, P. C. Kuo, A. Jamshidi-Roudbari, M. K. Hatalis, I. Biaggio, and A. T. Voutsas, Appl. Phys. Lett. 90, 151114 (2007).

${ }^{15}$ Dow chemical website, http://www.dow.com/cyclotene/prod/302246.htm.

${ }^{16}$ H. Aziz, Z. D. Popovic, and N.-X. Hu, Appl. Phys. Lett. 81, 370 (2002). 